\title{
Energy Efficient Routing Protocols for Mobile Ad hoc Networks: A Review
}

\author{
M. Izharul Hasan Ansari \\ Research Scholar \\ D/o Computer Sc. \& Engg. \\ NIMS University Jaipur
}

\author{
S.P. Singh, PhD \\ Associate Professor \\ D/o Computer Sc. \& Engg. \\ NIMS University Jaipur
}

\author{
M N Doja \\ Professor \\ D/o Computer Engineering \\ JMI University, N. Delhi
}

\begin{abstract}
As all the participating mobile nodes are mobile in nature in MANET and due to frequent movement of the participating mobile nodes, it is very difficult to predict the topology of the networks at any time. Which leads to frequent route failures and route discovery mechanism are required to activate very frequently, it causes a loss of significant amount of energy to perform the various operations among the participating mobile nodes of the networks. As all the participating mobile nodes are rely on limited consumable power supply. Therefore, energy management is one of the challenging tasks in MANET.
\end{abstract}

\section{General Terms}

MANET, Energy Efficient, Routing, Adaptive

\section{Keywords}

MANET, Transmission, Power, Energy, Routing

\section{INTRODUCTION}

The mobile ad hoc network (MANETs) is a collection of zero configurable mobile nodes without any physical infrastructure and centralized computing. All the participating mobile nodes are free to move without any prior information and permission. Due to the frequent movement of the participating mobile nodes the topology of the network is highly dynamic and unpredictable. Due to frequent movement of the mobile nodes in the network the available routes from one node to another nodes breaks very frequently. It leads to route failure and frequently route maintenance and route discovery process are required to activate very frequently, which causes a loss of significant amount of energy. As all the participating mobile nodes are rely on consumable and limited battery generated power supply for performing the various operations. Therefore, energy management is one of the challenging tasks in such type of networks.

The life time of the network can be extended by applying different energy management schemes. The basic aim of energy efficient routing protocols in MANETs is to reduce the energy consumption of the participating mobile nodes.

This review of literature presents the most important open issues, challenges and different techniques, which have already been developed by the various scientists to minimize the energy consumptions for increasing the life of the all participating mobile nodes of the networks to enhance the life span of the networks as a whole.

\section{CLASSIFICATION OF ENERGY MANAGEMENT SCHEMES}

The energy management schemes may be classified as follows:

- Battery management schemes
- Transmission power management schemes

- $\quad$ System power management schemes

These schemes [7, 2] further may be classified as follows

\subsection{Battery Management Schemes}

- Device Dependent Scheme

- Modeling and shaping of battery discharge pattern

- Battery scheduling

○ Data link Layer

- Lazy packet scheduling

- Battery Aware Medium Access Control Protocol (BAMAC)

○ Network layer

- Traffic Shaping

- Battery Energy Efficient Routing (BEE)

- Routing Based on Battery Status

\subsection{Transmission Power Management} Scheme

○ Data Link Layer

- Dynamic Power Adjustment

- Distributed Topology Control

- Distributed Power Control Loop

- Centralized Topology Control

○ Network layer

- Common Power (COMPOW)

- Globalized Power Aware Routing

- Localized Power Aware Routing

- Ad hoc Network Design Algorithm (ANDA)

- Determination of Critical Transmission Range

○ Higher Layer

- Congestion Control and Transmission Policies at TCP/IP Layer

- OS/Middleware approaches

\subsection{System Power Management Schemes}

- Device management schemes

- Power Saving Modes 
- Power Aware Multi-Access Protocol with Signaling (PAMS)

$\circ$ Processor power management schemes

- Low Power Design of Hardware

\subsection{System Power Management Schemes}

- Device Management Schemes

- $\quad$ Power Saving Modes

- Power Aware Multi-Access Protocol with Signaling (PAMS)

- $\quad$ Processor power management Schemes

- Low Power Design of Hardware

In [1], in this paper, the author has introduced a Minimum Energy Dynamic Source Routing Protocols (MEDSR) for Mobile Ad hoc Networks. The author in MEDSR has given two power levels, the first one is low power level route discovery and another one is high power level route discovery. At first stage all the nodes sends the route request packet to the next node with low power and he sets three attempts for discovering the route if could not find successfully the node changes it into high power level. It shows that sufficient amount of energy have been conserved and increases over all life time of the network. But the data packet travel through large number of hops to deliver the data at the destination node. Because of that delay per data packet have been increased significantly. The MEDSR may be modified and delay can be improved.

In [2], the author has proposed Common Power (COMPOW) for power control in mobile ad hoc networks. It says that excessively high power cannot be used to transmit the data packets from the source node to the destination node because of shared medium and it causes lot of interferences. This affects the traffic carrying capacity of the network and reduces the battery life. If network chooses low power for establishing the routes then it leads to route failure and route maintenance and route discovery process are required to activate very frequently, which causes a loss of significant amount of energy. Therefore, it shows that the network power level must be chosen neither too high to cause excessive interference which results in a reduced ability to carry traffic, nor too low to result in a disconnected network. This COMPOW have been designed and tested only for table driven routing protocols and apart from this compow technique is viable only for very dense network where number of participating mobile nodes is very high and covering area is small.

In [3], the author have studied the characteristics a of existing routing protocol AODV and observed that due to frequent movements of the nodes and dynamic topology of the network the AODV has the problem of fragile routes. To solve this problem, the author has proposed new route selection algorithms, i.e. AODV- Reliable Route Selection (AODV-RRS), to establish reliable routes. To achieve the desired goal they have used GPS information to obtain the mobile hosts' directions, speed and position of the node. They have also introduced stable zone and caution zone and these zones change dynamically depends upon the speed and direction of the participating mobile nodes. If the mobile nodes located very close to each other for long duration and maintain stable route then these nodes are said in stable zone and since the mobile nodes are far away from each other and are not able to make stable route between each other then these nodes are said in caution zone. The reliability of the links between the mobile nodes is decided by these zones. They found that the route selected by the proposed algorithm AODV-RRS lasts considerable longer than that of AODV. Therefore there is no need to activate the route maintenance and route discovery processes very frequently as in AODV because of that the energy consumption will be less and which helps to increase the overall life time of the networks.

In [4], the author has proposed slightly modified version of CMU Monarch Project's mobility - enhanced ns-2 simulator was used in conjunction with the model to analyze the energy consumption of the DSR, DSR-np and AODV routing protocols. The author has given the observations as:

Idle mode power is high and nodes are exposed to all traffic with in wireless range. The energy consumption of receiving and discarding the traffic is substantial and disproportional to the number of packets or bytes transmitted. Apart from these observations, it has been also observed that the cost of discarding traffic is low relative to idle mode power consumption; the cost of operating the interface in promiscuous mode is high. Wherever the node density of the participating nodes is high, the broadcast flooding is a very expensive affair. And the incremental cost associated with a packet is relatively small compared to the fixed costs. The number of packets has greater impacts on energy consumption than packet size does.

The author has observed that DSR is the most efficient of the three protocols in terms of packets sent and received. And DSR-np extracts topology information from source routing headers.

If the participating nodes acquire less network wide topology information and must initiate the route discovery process more frequently; this is seen in higher energy costs for broadcasting, and point to point route reply, route error messages traffic. Whereas AODV needs to initiate route discovery process very frequently, this gives much larger overhead energy cost than DSR-np at higher mobility levels though it is still less than DSR.

The variable transmission power in mobile ad-hoc routing protocols allowing nodes some control over their local node densities. And in addition to this, because energy spent at sender end as well at the end of receiver, it may prove to be an attractive QoS metric for network wide resource management and load balancing.

In [5], author has presented several routing techniques for energy constrained in mobile ad hoc networks and which are summarized as given below.

The basic idea has been given that those nodes should be avoided to consider in route which have least residual energy and have maximum chances to show disability for forward the data packet in the network during data forwarding.

In [6], the author has proposed the shortest hop routing technique until the nodes consume a predefined part of their energy and then the routing algorithm switch to an algorithm with the power aware matrices, which has been presented in [7]. The behavior of a conditional Maximum - Minimum Battery Cost Routing (MMBCR), 
(CMMBCR) routing algorithm is in function of the battery capacity of the nodes.

In [8], the author has proposed an idea of knowing the life time of the node. The route of the traffic can be diverted if needed to avoid the nodes failure due to the in sufficient amount of energy. Kim et al in [9] has proposed a new routing metric for predicting battery lifetime, i.e. the energy drain rate, is computed by exponential weighted moving average method and gives the estimated energy consumption per second. The cost function is defined as the ratio between the residual battery power (RB ) and drain rate $(\mathrm{DR})$ at a node:

$C=R B P / D R$. The Minimum Drain Rate (MDR) mechanism is a max-min algorithm (an analogue to MMBCR) which implements the above cost function. In [8], the author has extended the MDR algorithm to Conditional MDR, which shares the same objective and principles with CMMBR.

During route discovery process all the participating nodes of the networks have participated in the process while the final decision has been made at source or destination node.

In [10], the author has given the right to all the participating nodes of the network to decide that they want to participate in route discovery process or not. In local Energy aware Routing (LEAR), the participating node has to make the decision about the willingness of intermediate node to respond the route request and forward the data traffic further. This technique has successfully shifted the responsibility for reacting to changes in the energy budget of the node from the source-destination node to the intermediate nodes leads to avoid the need of periodically exchanging control information.

In [11], the author has proposed Residual Packet Capacity (MRPC) algorithm this algorithm used residual battery energy as main factor in route selection decision but apart from this also added the expected energy spent metric, which is main contribution of the author. The link characteristics mainly affect the energy consumption and can lead to excessive re-transmission of the packets. The cost of choosing a specific link is defined as the maximum number of packets that can be transmitted by the transmitting nodes over the specific link assuming the complete absence of the any other cross traffic at that node [11]. The maximum life time of a given route is determined by the weakest intermediate node.

In [12] and [13], the author has studied the lifetime of the battery based on recovery of the power and over consumed energy, experienced when no/new traffic is transmitted design a cost function which reflects the energy consumption. The function in [5] is minimum function over the cost function of all routes. However in [30], the sensitivity of the algorithm has been proposed towards the battery behavior makes it switch between different available paths so that the battery recovery can take place and the maximum of the node's battery capacity can be attained.

In [14], the author has proposed a technique for improving the delay caused due to the link failure resulting activates the route discovery. In this technique, the power levels of the participating nodes have been computed. During the transmission of the traffic if the power level of specific nodes goes down to a certain thresh hold value then it sends the warning message to the predecessor of the node for determining an alternative route for forwarding the data packets towards the destination node. The performance of the DSR protocol under preemptive routing has been evaluated in [15]. The signal strength thresh hold is used to generate the warning message to the predecessor node for discovering the alternative route for delivering the data packets successfully. And this is based on the concept of preemptive region which is given in [16].

In [17], the author has proposed a technique to enhance the power based routing protocol. In this technique if the signal strength goes down below a certain threshold value, generates a warning message being sent to the source node. And the source node has multiple paths to destination found during initial process route discovery.

The [18] proposed the preemptive multipath ad hoc on demand distance vector routing (PMAODV) based on the concept of AODV with multipath routing and preemptive routing protocol.

In [19], the author has been proposed the improvement in AODV by adding the concept of preemptive routing and also propped the predictive preemptive ADOV (PPAODV) routing techniques. Whenever the mobile node goes to the preemptive region then the strength of the signals goes down a certain threshold. For sending the prediction when to send the warning message it uses three consecutive signal strength of the packets received from the predecessor in Lagrange's interpolation formula to compute the strength of the received signals strength P. when $\mathrm{P}$ goes down a certain threshold value, a warning message is sent to the predecessor and the predecessor node starts to repair local route by finding the alternate route to the destination node for smooth data traffic.

In [20] the author has proposed cache enabled preemptive Dynamic Source Routing (CPDSR) technique. This technique has suggested a solution by using old routes available in the nodes cache. As preemptive routing technique produces early warning so route error (RERR) packets are not being broadcasted hence nodes are still with that old routes information in their cache. The source nodes receives a warning message from the warning node then source node refreshes its local route cache and tries to trace the new route in to its cache to the destination node if exists then uses that route otherwise broadcasts the route request (RREQ) packets with unstable link information to find the new routes to the destination. Unstable link information is kept in RREQ because all the participating nodes on the route refresh their caches with this and the new replied routes do not contain such unstable links. When the source node sends RERR message to the warning node so that all the nodes on this route refreshes their cache and warning node must know that the route has been changed. The author has also proposed a method to determine reaction time. It is the time to determine when a link is regarded as unstable.

In [21], the author has proposed a technique to hand off the router in case of strength of the link is weak. It is determined by considering the power difference table and neighbor power list which is kept by all the participating node of the networks. Based on these table values warning nodes determines neighbor node which is reachable from source and destination node both so that the data can be diverted through that particular path and the warning node broadcast a hand off packet containing the address of the predecessor and successor to make the route. If the 
neighbor node with sufficient link stability cannot be determined then allow the link fail to occur and later route repair is done as per AODV protocols standard mechanism.

[22] In this research paper, the author has derived an asymptotic expression for the computation of the average variable-range transmission and traffic capacity in wireless ad hoc networks. They have also shown that the use of a variable-range transmission based routing protocol uses lower transmission power and increases capacity compared with common-range transmission approaches. The authors also derived expressions for the route discovery and maintenance phases of an ideal on-demand routing protocol. It also shows that there is an optimum setting for the transmission range, not necessarily the minimum, which maximizes the capacity available to nodes in the presence of node mobility.

In [23] this research paper, the investigators have tried to investigate the impact of variable transmission range on power saving in MANETs. The performance analysis was done on two routing protocols: Minimum Hop Routing (MHR) and Minimum Total Power Routing (MTPR). After analyzing the results of their work, the authors has made following conclusions as given below:

- Power saving of MTPR is always higher than MHR, It indicates to increase the percentage power saving MTPR protocol should be used rather than MHR.

- Power consumption of MHR is higher than MTPR because MHR takes a path that tries to minimize number of nodes to reach to destination. On the other hand MTPR tries to select a path that tries to minimize the total power from source to destination.

Above analysis shows that variable transmission range has the potential to increase the power savings in mobile ad hoc networks and hence the overall lifetime of the networks.

In this paper the authors has presented a strong reason for the use of variable transmission range in MANET.

In [24] this paper, the authors presented a technique called efficient power aware routing (EPAR) which is basically an improvement on DSR. This study has evaluated three power-aware ad hoc routing protocols in different network environment taking into consideration network lifetime and packet delivery ratio. Overall, the finding shows that the energy consumption and throughput in small size networks did not reveal any significant differences. However, for medium and large ad-hoc networks the DSR performance proved to be in-efficient in this study. In particular, the performance of EPAR, minimum total transmission power routing (MTPR) and DSR in small size networks was comparable. But in medium and large size networks, the EPAR and MTPR produced good results and the performance of EPAR in terms of throughput is good in all the scenarios that have been investigated. The EPAR algorithm outperforms the original DSR algorithm by $65 \%$.

In [25] this paper, the analysis shows that the optimum transmission radius is influenced more by the node density than the network coverage area. It is observed that when the path-loss exponent is four, the optimal transmission ranges are almost identical over the range of node densities that they studied. However, the optimal transmission range decreases noticeably as the node density increases when the path-loss exponent is only two.

A node can effectively increase its transmission range by reducing the data rate without changing its transmission power, contrary to fixing the data rate and adapting the transmission range by dynamically adjusting the transmission power. It would be interesting to consider such a data-rate adaptive possibility to conserve energy.

Furthermore, the interference among multiple traffic flows may affect the transmission range optimization by introducing packet collisions and re-transmissions.

In [26] this paper, a fuzzy adaptive transmission range and fuzzy based threshold energy for location aided routing protocol has been proposed by the authors. In this proposed protocol, the energy of a mobile node is conserved by employing fuzzy adaptive transmission power control depending on the minimum number of neighboring nodes to maintain network connectivity and power aware routing based on fuzzy threshold energy. The experimental results of the paper show that the proposed protocol Fuzzy Adaptive Transmission Range Based Power Aware Location Aided Routing (FTRPALAR) performs better in terms of average energy consumption and network lifetime as compared to the LAR and variable transmission range power aware location aided routing (VTRPALAR) routing protocols. The proposed FTRPALAR is able to achieve $18 \%$ more lifetimes compared to VTRPALAR.

In [27] Topology adaptive clustering algorithm (TACA) is energy efficient and topology adaptive distributed clustering algorithm that ensures better cluster stability and enhances the network life time. Being a topology adaptive algorithm, it eliminates the freezing time of motion of mobile nodes during the cluster setup. A node with lower mobility and higher available battery power is chosen for cluster head so that cluster stability is improved. Introducing the selection of non-volunteer nodes reduces the number of global re-elections for the cluster heads. At the same time the load on nodes acting as heads is also minimized enhancing the network life time. But TACA results in slightly higher cluster population that increases the number of members in the virtual back bone of communication. To further improve the clustering results, a transmission range adjustment protocol has been designed that enables a node to adjust its transmission range as and when required. The new design helps in reducing the number of isolated cluster heads and eventually the number of hops in the back bone. It is seen that when the transmission range is adjusted for certain nodes, the clustering algorithm reduces the density of clusters as well as the number of re-elections in the network without compromising the network life time.

In [28] this paper, the authors have provided new insights beyond the literature that strongly support the development of new variable-range transmission-based routing protocols. Their results indicate that a variable-range transmission approach can outperform a common-range transmission approach in terms of power savings and increased capacity. They have derived an asymptotic expression for the computation of the average variablerange transmission and traffic capacity in wireless ad hoc networks. They have shown in this research paper that the use of a variable-range transmission-based routing protocol uses lower transmission power and increases 
capacity compared with common-range transmission approaches.

\section{OTHER APPROACHES}

Generalized Salvaging Mechanism (GSM-DSR) uses two optimization techniques called "Generalized salvaging mechanism and cache update of intermediate nodes. GSM search for an alternative route in upstream nodes cache. Localized route repair (LRR) scheme can be implemented on any on demand routing protocols. LRR defines a neighbor node as a node, which is on the route and is in the instant neighborhood of the moved node. The approach repairs the broken link locally. Repaired backup approach tackles link failure by backup routes and these routes are generated from the destination node to the source node along with the best route during route discovery.

In [29] MEDSR (Minimum energy dynamic source routing) and HMEDSR (Hierarchical minimum energy dynamic source routing) protocols are proposed. The MEDSR uses different power levels (two) during route discovery process to identify low energy paths. After finding the path, the transmit power levels of the nodes along those routes are adjusted link by link to the minimum required level. But it uses flooding during route discovery process resulting overhead in large networks. This affects routing performance severely. Although overhead packets are not in large number but consume significant amount of energy. So to overcome this (routing control and MAC packets) HMEDSR is proposed. It is the combination of HDSR (hierarchical dynamic source routing) and MEDSR. HDSR helps in reducing overhead packets and MEDSR saves energy in transmitting data packets. Author has not tested the influence of mobility on the proposed protocols.

In [30] the author quantitatively measures the inaccuracy of state information of nodes in optimized link state routing (OLSR) routing protocol. State information may be such as residual energy level, queue length and link attribute such as bandwidth, delay and error rate etc. A node may have outdated information about the residual energy of other nodes. So the author measured the inaccuracy present and also proposed two approaches to improve inaccuracy prediction and smart prediction. In prediction last two values are taken to determine the consumption rate of other nodes at a particular instant of time. And smart prediction is applied in case information about other nodes is absent. So its residual energy value is predicted by taking the average consumption rate of others (if present) or itself.

In [31] author has proposed new cost function for determining the cost of the route from source to destination. The cost function includes many variables like unstable_nodes_count (a), number_of_neighbors (b) and buffered packets (c). These variables are added to route request packet in addition to amount of data source wants to send. During route discovery intermediate nodes adds their values of these variables to the existing values in route request packet. Over the route, nodes have different number of neighbors, buffered packets and different number of unstable nodes. A node is called stable if its certain number of neighbors (40\%) does not change during a fixed time interval (say 3 seconds). The destination receives the route request with cost function value calculated for the traveled path and stores it in the buffer for the fixed time. If in the mean time another route request comes with smaller cost function value then previous route request is discarded. Otherwise wait for the fixed time to expire and retrieve the stored route request and send the route reply. In it the nodes over the path with more number of neighbors are discouraged as they will do overhearing and will loss energy soon. Similarly path with stable nodes is encouraged otherwise path breaks are likely to occur and cause maintenance phase to start will result in energy loss. In continuation to this nodes with buffer queues cause timer expired causing retransmission resulting in energy loss. In the technique a node forwards the route request if nodes remaining lifetime is more than the needed to send the packet. Otherwise it drops the request packet. The nodes lifetime is calculated by its residual energy and drain rate.

In [32] the author has proposed the method to minimize broadcasting overhead. Three different techniques are being used namely flooding, probability based flooding and clustering. The flooding is used to find the location information of nodes and clustering (K Mean algorithm) is used to minimize overhead which in turn will result in energy efficiency. Several other techniques have proposed to improve the performance of on demand routing protocols in case of link failures such as backup source routing, modification of DSR protocol, packet received time and link expiration time etc.

In [33] Ad hoc backup node setup protocols had been proposed to improve DSR performance in case of link failure. This is achieved by adding backup routes. But the backup routes may have changed due to topological changes by the time is required as an alternative means in case of a link failure in the primary route. And the changes in the topology are not reflected in backup route. Way point routing protocol (WPR) divides the network into segments through waypoints (some intermediates nodes) and DSR is used for inter segment and AODV for intra-segment routing. WPR suffers significant overhead to re-establish a new global route discovery.

\section{CONCLUSION}

In this paper a comprehensive literature review has been done, which presents the different facets of energy saving techniques to increase the life span of the networks. The challenging aspects of the problem have been revealed by surveying the various relevant issues concerning the optimization of the energy consumption in MANET. The researchers may put their efforts to improve the delay, overhearing, lifetime of the stable routes, and may also try to develop a technique for adaptive transmission range for the participating mobile nodes of the networks. This review paper will help the research scholars to identify the open research issues in the concerned field and also attract the other researchers towards this important research issue.

\section{ACKNOWLEDGMENT}

I am very grateful to my supervisors, who have inspired and helped me a lot to bring all the material into a form of this review paper.

\section{REFERENCES}

[1] Mohammed Tarique and Roman Islam, "Minimum Energy Dynamic Source Routing Protocols for Mobile Ad hoc Networks" International Journal of Computer Science and Network Security Vol. 7 No. 11, 2007, pp 304-311 
[2] Swetha Narayanaswamy, Vikas Kawadia, R. S. Sreenivas and P. R. Kumar, "The COMPOW protocol for power control in ad hoc networks: Theory, architecture, algorithm, Implementation and experimentation" http://citeseerx.ist.psu.edu pp 1-20

[3] Won-Ik Kim, Dong-Hee Kwon, Young-Joo Suh, “A Reliable Route Selection Algorithm Using Global Positioning Systems in Mobile Ad-hoc Networks" IEEE International conference provceedings, Vol 10, June 11-14, 2001, pp-3191-3195, Helsinki.

[4] Laura Marie Feeney, "An Energy Consumption Model for Performance Analysis of Routing Protocols for Mobile Ad Hoc Networks" Mobile Networks and Applications, Kluwer Academic Publishers, Vol. 6, 2001, pp 239-249.

[5] Natalia Vassileva, Francisco Barcelo-Arroyo, "A Survey of Routing Protocols for Energy Constrained Ad Hoc Wireless Networks" IEEE International Conference proceedings, Vol. 1, Decemebr 6-8, 2007, pp 522-527.

[6] S. Singh, M. Woo, C. S. Raghavendra, "Power-aware routing in mobile ad hoc networks", in Proc. 4th ACM/IEEE MobiCom, 1998, pp. 181 - 190.

[7] C.K. Toh, "Maximum battery life routing to support ubiquitous mobile computing in wireless ad hoc networks", IEEE Comm. Mag., June 2001, vol.39, issue6, pp. $138-147$.

[8] D. Kim, G.L. Aceves, K. Obraczka, J -C. Cano, P. Manzoni, "Routing mechanisms for mobile ad hoc networks based on the energy drain rate", in IEEE Trans. Mobile Computing, April - June 2003, vol. 2, no. 2 , pp. $161-173$.

[9] B. C. Kim, J. Y. Lee, H. S. Lee, J. S. Ma, “An ad-hoc routing with minimum contention time and load balancing”, in Proc. IEEE GLOBECOM, 2003, pp. 81 -85 .

[10] S. Yin, X. Lin, "Multipath minimum energy routing in ad hoc network", in Proc. ICC, 2005, pp. 3182 3186.

[11] A. Misra and S. Banerjee, "MRPC: Maximizing network lifetime for reliable routing in wireless environments", in Proc. IEEE WCNC, 2002, pp. 800 -806 .

[12] C.-F. Chiasserini and R. R: Rao, "Routing protocols to maximize battery efficiency", in Proc. IEEE MILCOM, 2000, pp. 496 - 500 .

[13] X. Zheng, W. Guo, R. Liu, Y. Tian, “A new dynamic load-aware based load-balanced routing for ad hoc networks", in Proc. IEEE ICCCAS, 2004, pp. 407 411.

[14] Sharvani G S, Dr. T.M. Rangaswamy, Nabendu Chaki et al., "Efficient packet delivery approach for adhoc wireless networks," (Eds.): NeTCoM 2010,CSCP 01, 2011, pp. 42-49, 2011.

[15] B R Sujatha, M V Satyanarayana, "Improved network connectivity in MANETs," International Journal of Computer Networks \& Communications (IJCNC), vol.I, no.3, October 2009.
[16] Tom Goff, Nael B. Abu-Ghazaleh, Dhananjay S. Phatakand Ridvan Kahvecioglu,"Preemptive Routing in AdHoc Networks," ACM SIGMOBILE 7/01 Rome, Italy, June 2002.

[17] Sujatha P. Terdal, V. D Mytri, and A Damodaram, "A Preemptive Multipath Routing Protocol for Mobile Ad Hoc Networks," International Journal of Computer Science and Communication Technologies, vol. II, no. 1, July 2009

[18] Manoj Kumar Singh, Brajesh Kumar, Chiranjeev Kumar and Manish Gupta, "Preemptive MultipathAdhoc on Demand Distance Vector Routing Protocol," International Journal of Computer Science and Information Technology, vol I, no.1, Jan 2011 , pp. $36-40$.

[19] Sofiane Boukli Hacene, Ahmed Lehireche, Ahmed Meddahi, "Predictive Preemptive Ad Hoc On Demand Distance Vector Routing," Malaysian Journal of Computer Science, Vol. 19 (2), 2006.

[20] Wenbo Zhu, Xinming Zhang, Yongzhen Liu, Nana Li, "Improve Preemptive Routing Performance in Mobile Ad hoc Networks with Cache-enabled Method," Proc. of International Conference on Communications and Networking in China (Chinacom), August 2008.

[21] Srinath Perur, Abhilash P. and Sridhar Iyer, "Router Handoff: a preemptive route repair strategy for AODV," proc. of the IEEE International Conference on Personal Wireless Communications, New Delhi, India, 15-17 December 2002, pp.168-171.

[22] Javier Gomez and Andrew T. Campbell, "A Case for Variable-Range Transmission Power Control in Wireless Multihop Networks" IEEE INFOCOM 2004

[23] C.K Nagpal, Maninder Kaur,Shailender Gupta and Bharat Bhushan, "Impact of variable transmission range on manet performance" International Journal of Ad hoc, Sensor \& Ubiquitous Computing (IJASUC) Vol.2, No.4, December 2011

[24] Shivashankar, Suresh H.N, Varaprasad Golla, Jayanthi G, "Designing Energy Routing Protocol with Power Consumption Optimization in MANET" IEEE Transactions on Emerging Topics in Computing, 2013.

[25] Jing Deng, Yunghsiang S. Han, Po-Ning Chen, and Pramod K. Varshney, "Optimal Transmission Range for Wireless Ad Hoc Networks Based on Energy Efficiency" IEEE transactions on communications, vol. 55, NO. 9, pp 1772-1782, September 2007

[26] P.S.Hiremath and Shriharim.Joshi, "Fuzzy Adaptive Transmission Range based Power Aware Location Aided Routing", International Conference on Information and Communication Technologies (ICICT-2014)

[27] Suchismita Chinara and Santanu Kumar Rath, "Topology Control by Transmission Range Adjustment Protocol for Clustered Mobile Ad Hoc Networks, ISRN Communications and Networking, Hindawi, 2010

[28] Javier Gomez and Andrew T. Campbell, "Using Variable-Range Transmission Power Control in 
Wireless Ad Hoc Networks, IEEE TRANSACTIONS ON MOBILE COMPUTING, VOL. 6, NO. 1, JANUARY 2007

[29] Mohammed. Tarique, Kemal. E. Tape, "Minimum Energy Hierarchical Dynamic Source Routing for Mobile Ad Hoc Networks," Elsevier Science Publisher, Netherland, vol. VII, no. VI, Aug 2009, pp.1125-1135.

[30] Thomas Kunz and Rana Alhalimi, "Energy efficient proactive routing in MANET: Energy metrics accuracy," Journal of Ad Hoc Networks, vol. VIII, no.VII, Elsevier Science, pp.755-766.

[31] Mehdi Lotfi and Sam Jabbehdari and Majid Asadi Shahmirzadi, "A New Energy Efficient Routing Algorithm Based on a New Cost Function in Wireless Ad hoc Networks," Computing Research Repository, vol. Abs/1006.4, 2010

[32] N. Karthikeyan and V. Palanisamy and K. Duraiswamy, "Reducing broadcast overhead using clustering based broadcast mechanism in Mobile Ad Hoc Network,” Journal of Computer Science, 2009. 